\title{
Paranoid Personality Disorder
}

National Cancer Institute

\section{Source}

National Cancer Institute. Paranoid Personality Disorder. NCI Thesaurus. Code C92630.

A disorder characterized by an enduring pattern of behavior based on the pervasive belief that the motives of others are malevolent and that they should not be trusted. 